\title{
Actualización en los puntos clave de la endometriosis
}

Updates on the highlights of endometriosis

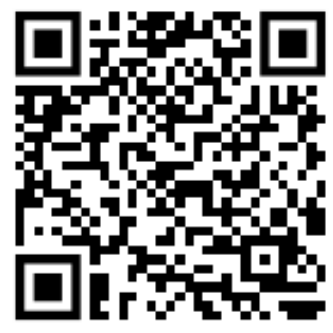

${ }^{1}$ Dra. Nathaniela María Ulett Araya
Hospital La Católica, San José, Costa Rica
$\underline{\text { nat.ulett@gmail.com }}$
(i) https://orcid.org/0000-0001-7769-3288

RECIBIDO

CORREGIDO

ACEPTADO

$15 / 03 / 2019$

$28 / 03 / 2019$

$10 / 04 / 2019$

\section{RESUMEN}

La endometriosis es un desorden ginecológico caracterizado por el desarrollo de tejido endometrial fuera del útero. Es una enfermedad común, que induce una respuesta inflamatoria que más adelante podrá mediar una gran gama de síntomas, entre los que comunmente se destacan, dolor e infertilidad. Se desconoce la causa exacta de la endometriosis, pero se sabe que hay factores de riesgo conocidos para su aparición, como la menarca más temprana y la base genética que expone mayor riesgo con familiares de primer grado afectados. Sobre la etiología, se habla mayormente sobre la teoría de la menstruación retrógrada, la teoría de la metaplasia celómica y la de diseminación linfática o vascular. Para establecer el diagnóstico, la laparoscopía diagnóstica se continúa visualizando como el estándar de oro, aunque hay muchos otros métodos de imagen que nos pueden ser de gran utilidad en la evaluación. Finalmente, para ofrecer un tratamiento adecuado a las pacientes, a pesar de que cada día aparecen más opciones, es necesario individualizar la elección según las características, tolerabilidad y necesidades de cada una. Por lo anterior, existen diferentes tratamientos como: no hormonales, medicamentos hormonales convencionales, quirúrgicos y los emergentes.

PALABRAS CLAVES: dolor pélvico; dismenorrea; endometrio; estrógenos; infertilidad.

${ }^{1}$ Médico general, graduada de la Universidad de Ciencias Médicas (UCIMED). Código médico:15237 nat.ulett@gmail.com

\section{ABSTRACT}

Endometriosis is a gynecological disorder characterized by the development of endometrial tissue outside of the uterus. It is a common disease, which induces an inflammatory response that can later mediate 
a wide range of symptoms, among which there commonly stands out, pain and infertility. The exact cause of endometriosis is unknown, but yet there are some known risk factors for its outcome, such as the earlier menarche and the genetic basis that exposes greater risk with affected first-degree relatives. Regarding the etiology, there is more to talk about the theory of retrograde menstruation, the theory of coelomic metaplasia and the lymphatic or vascular dissemination. To establish the diagnosis, the diagnostic laparoscopy is still visualized as the gold standard, although there are many other imaging methods that can be really useful during the evaluation. Finally, to offer an appropriate treatment to the patients, despite the fact that more options come up every day, the choice must be individually customized according to the characteristics, tolerance and needs of each patient. For the above, there are different treatments such as: non-hormonal, conventional hormonal drugs, surgical and emerging.

KEYWORDS: dysmenorrhea, endometrium; estrogens; pelvic pain; infertility.

\section{INTRODUCCIÓN}

La endometriosis es una patología ginecológica frecuente, crónica, inflamatoria, estrógeno-dependiente, que se caracteriza por la proliferación de glándulas endometriales funcionales y estroma fuera de la cavidad uterina (Asghari S, 2018) (Hogg S, 2018) (Patel $B, 2018)$. Este tejido ectópico induce al sangrado, la inflamación crónica y la fibrosis, que resultan en la formación de nódulos endometriales y adhesiones (Tomassetti C, 2018). Asimismo, se presenta resistencia a la progesterona y reducción general de sus receptores (Clemenza S, 2018). Todo esto posteriormente mediará el desarrollo de la sintomatología.

Las localizaciones más frecuentes son el peritoneo pélvico (endometriosis superficial), el ovario; con una frecuencia de $31-44 \%$ en todo el conjunto de las mujeres (en su mayoría presentándose como endometriomas) (Chauvet $P, 2017$ ) $\mathrm{y}$ el espacio retroperitoneal, pudiendo provocar lesiones parietales de los órganos pélvicos.

El objetivo del presente artículo de revisión es dar a conocer de manera integral una de las patologías más frecuentes de las mujeres en nuestro medio, pero con muchos desafíos; desde la comprensión de su fisiopatología hasta la prescripción del tratamiento adecuado para cada caso. Consiste en darle énfasis a los conceptos más novedosos hasta el momento y que explican de manera más precisa cada tópico, para así comprender mejor la enfermedad.

\section{EPIDEMIOLOGÍA}

La medición precisa de la prevalencia e incidencia es difícil, debido a que se requiere de la visualización quirúrgica para realizar un diagnóstico definitivo de la enfermedad $(4,7)$. Pese a esto, la prevalencia se estima entre un 2 a $10 \%$ en 
mujeres en edad fértil y hasta en un 35$50 \%$ de las mujeres que experimentan dolor o infertilidad $(1,8)$. El pico de incidencia usualmente se presenta entre los 25 y 35 años de edad, aunque también puede afectar a mujeres más jóvenes o hasta posmenopáusicas que presenten dolor pélvico.

Para su estudio, se ha propuesto la clasificación de la endometriosis en tres subgrupos o fenotipos, que son: endometriosis superficial peritoneal, endometriomas ovaricos y endometriosis profunda (9). Se habla de esta última estancia cuando se produce una invaginación bajo el peritoneo, que supere los $5 \mathrm{~mm}$ de profundidad (10). La endometriosis profunda, precisamente afecta al $1 \%$ de las mujeres en edad reproductiva y se considera de las formas más severas e incapacitantes de esta enfermedad. Puede llegar a comprometer desde el septo recto vaginal y los ligamentos uterosacros, hasta el fondo de saco vaginal posterior, recto y estructuras abdominales como uréteres, intestinos y vejiga; más comúnmente $(9,11)$.

\section{FACTORES DE RIESGO}

Para A pesar de que no se conoce con exactitud la causa de la endometriosis, sí se sabe que hay varios aspectos que pueden influir en su aparición. La tendencia actual hacia la menarca cada vez más temprana y el retraso en la edad de inicio de la paridad, ha ocasionado un mayor número de ciclos menstruales y ovulaciones y por lo tanto exposición prolongada a estrógenos endógenos y a mayor cantidad de menstruaciones retrógradas (se comentará más adelante); incrementando el riesgo de endometriosis $(7,3)$. Los ciclos menstruales más cortos (menores a 26 días), que se presentan especialmente en la adolescencia; y el bajo peso al nacer, también se han asociado con tasas más altas de endometriosis (7).

Además existe un componente genético que se ha investigado y se ha estimado en un $51 \%$ sus probabilidades de ser heredado. Se habla con mayor frecuencia de un locus en el cromosoma 7p15.2 que se ha ligado con endometriosis en mujeres de ascendencia Europea, que se asocia más a la enfermedad moderadasevera (2). También existe una base genética, que expone mayor riesgo de endometriosis a aquellas mujeres con familiares de primer grado afectados, con un aumento en la prevalencia de un 6 a un $9 \%$ y hasta $15 \%$ para la presentación de enfermedad severa (12). De manera similar, se han hecho observaciones de concordancia de endometriosis en gemelas, en las que se ha encontrado que la edad de aparición de los síntomas y la prevalencia de la endometriosis son bastante similares (2).

Así, en varias ocasiones se ha lanzado el supuesto de que la dioxina y la exposición completa a la radiación pueden tener efectos genómicos y epigeneticos en relación con el desarrollo de la endometriosis $(12,11)$. Se ha especulado mucho sobre este tema, pero aún no se tiene un panorama bien claro.

Se ha visto que la etnia tiene un papel importante en los factores de riesgo, pues en las mujeres Asiáticas es más prevalente la endometriosis, mientras que en las mujeres Africanas se presenta con menor frecuencia, en comparación con las mujeres caucásicas (2).

Asimismo, hay estudios que muestran que la exposición al dietilestilbestrol (DES) in útero influencia potencialmente 
la aparición de la endometriosis al alterar la exposición de los receptores estrogénicos, aumentar la menstruación retrógrada y causar disfunción del sistema inmune (7).

La lactancia prolongada y los embarazos se consideran factores protectores; así como hay algunos estudios que arrojan que el consumo de verduras, frutas y ácidos grasos omega-3 de cadena larga se asocian con disminución del riesgo de endometriosis, pero falta más evidencia para afirmarlo $(7,3)$.

\section{ETIOLOGÍA}

Hay muchas teorías sobre la etiología de la endometriosis, pero aún no hay un mecanismo definido y acordado. La teoría más utilizada y popular para explicar las lesiones o implantes peritoneales es la Teoría del Transplante Retrógrado de Sampson, también conocida como teoría de la implantación o de la menstruación retrógrada. En esta se expone que las células del endometrio se conducen por las trompas de Falopio en dirección retrógrada, mediante reflujo, durante la menstruación, llegan hasta la pelvis y se instalan en las superficies serosas de esta $(1,2)$. La evidencia que apoya esta teoría se basa en que las mujeres afectadas usualmente presentan mayores cantidades de sangre en reflujo durante las menstruaciones, en comparación con las que no presentan endometriosis (3). Pero se sabe que hasta $90 \%$ de las mujeres pueden tener menstruación retrógrada; y solo el $15 \%$ de estas tiene endometriosis confirmada (2). Por lo que, esta incongruencia asociada a la presentación de endometriosis en sitios extra pélvicos, nos manda a considerar otras teorías.

La teoría de la metaplasia celómica o la teoría de Robert Meyer, consiste en una transformación metaplásica de las células que recubren el peritoneo visceralabdominal; en tejido endometrial, por estímulo hormono-ambiental (1-3). Ha tenido aceptación para casos de endometriosis en ausencia de menstruación, como en mujeres premenopáusicas y posmenopáusicas.

Otra de las teorías que se analiza es la teoría embrionaria Mulleriana, donde se propone que la endometriosis se basa en una alteración de las estructuras del tracto genital durante la organogénesis; y esto permite que haya tejido endometrial fuera de la cavidad uterina, expresando estrógenos y CA-125 $(2,3)$.

La teoría de la diseminación vascular o linfática explica los implantes de endometriosis en sitios extra pélvicos, como nódulos linfaticos abdominales, pulmón, pleura y cerebro, pero falla en que no explica las lesiones pélvicas comunes (3). Si se habla de trasplante directo, se puede evidenciar en alguna intervención quirúrgica pélvica, cuando se descubre endometriosis en cicatrices abdominales.

Asimismo, se han planteado otras hipótesis reportadas en la literatura. Por ejemplo, la teoría de las células madre; que se dice que se trata de células indiferenciadas con la capacidad de regenerar en depósitos endometriales (3), la teoría de la disfunción inmune; en la que por enfermedad autoinmune hay mayor inflamación y eliminación deficiente de desechos de menstruación, con promoción del endometrio ectópico, entre otras (2). 
Es probable que la causa sea una combinación de estas teorías.

\section{PRESENTACIÓN CLÍNICA}

El dolor en forma de dismenorrea secundaria, dolor pélvico generalizado y dispareunia; es de los síntomas más comunes $(1,2,11)$. Incluso se podría decir que el dolor pélvico cíclico y la infertilidad son los síntomas clásicos de la endometriosis. La dismenorrea secundaria comienza generalmente de 36 a 48 horas antes del inicio de la menstruación; y se puede presentar como dolor sordo o dolor pélvico severo, de manera unilateral o bilateral. Además puede irradiarse a la espalda baja, las piernas y la ingle. Se acompaña de incapacidad para cumplir con las actividades de la vida diaria (absentismo profesional o académico) y muchas veces obliga a solicitar asistencia a los servicios de urgencias pues los analgésicos de primer nivel no lo alivian (11). Se presenta sangrado uterino anormal en 15 a $20 \%$ de las pacientes con endometriosis $y$ usualmente es en la forma de manchado premenstrual 0 menorragia. La dispareunia traduce una afectación de los ligamentos uterosacros, muchas veces por endometriosis profunda. Con menor frecuencia, pero de igual manera problemáticos, son los síntomas relacionados con el aparato gastrointestinal y urinario, como el dolor abdominal cíclico, diarrea o estreñimiento intermitente, poliuria, disuria, hematuria, entre otras $(1,2,11)$. Se estima que aproximadamente un $20 \%$ de las mujeres con endometriosis presentan de manera conjunta, síndrome del intestino irritable, migrañas y cistitis (2). Otra de las manifestaciones, aunque menos frecuente, es el neumotórax espontáneo recurrente o también llamado catamenial, que se presenta típicamente durante la menstruación cuando hay implantes endometriales en pleura (11).

La mayoría del tiempo se toma entre 5 a 10 años desde el inicio de los síntomas hasta el momento del diagnóstico confirmado de endometriosis; con una media establecida en 7,5 años $(1,2,11,13)$. El retraso puede deberse a que muchas mujeres minimizan la gravedad de sus síntomas y deciden consultar y manifestarlos hasta que la infertilidad comienza a afectarles. En muchas ocasiones también puede deberse al desafío de diferenciar las causas de la dismenorrea, utilizando tratamiento empírico por largos períodos con respuesta parcial; así como algunos otros casos de mujeres que fueron sometidas a laparoscopías a edades muy tempranas o durante los inicios de la enfermedad y en las que no se reportó endometriosis; sin embargo pudo haber sido precoz para expresarse completamente en dicho momento (2).

Además de una historia clínica dirigida, es muy importante la exploración física, con examen abdominal y pélvico. Se deben identificar algunos signos que se asocian con enfermedad profunda, estos incluyen la hipersensibilidad al movimiento uterino, movilidad disminuida de los órganos pélvicos, nódulos sensibles en el fórnix posterior, engrosamiento palpable de los ligamentos uterosacros o desplazamiento lateral del cérvix; así como podría tratarse de algún endometrioma, que sería palpable como una masa anexial (2). A la especuloscopía, ocasionalmente se pueden notar lesiones azuladas en mucosa vaginal o en el cérvix. Ante cualquier hallazgo sugestivo de 
enfermedad profunda se deben completar estudios y referir a la paciente a un centro de primer 0 segundo nivel, con especialistas para continuar manejo.

\section{DIAGNÓSTICO}

Hay muchas vías por las que se puede diagnosticar o fallar en el diagnóstico de la endometriosis. Algunas mujeres se diagnostican por dolor pélvico, dismenorrea o dispareunia; mientras que otras se diagnostican durante la evaluación por infertilidad, o bien, de manera incidental mediante la observación directa de las lesiones durante procedimientos quirúrgicos pélvicos no relacionados (7).

No es difícil cometer errores si se intenta establecer un diagnóstico a partir de la sintomatología de la paciente. Incluso se sabe que la gravedad de la enfermedad no está relacionada con el número y la intensidad de los síntomas, pues las mujeres con enfermedad avanzada podrían tener molestias escasas o nulas, mientras que las que presentan enfermedad mínima o leve pueden llegar a presentarse con dolores incapacitantes (11).

Los métodos de estudio con imágenes son una herramienta que puede ser útil en algunos casos para la evaluación de la endometriosis y su extensión. La ecografía pélvica es el método de primera línea para la búsqueda de una endometriosis pélvica ante la sospecha, se debe efectuar por vía supra púbica y endovaginal $(2,10,11)$. Permite identificar endometriomas ováricos y se puede utilizar además antes de decidir un método quirúrgico; presenta la ventaja de ser de los instrumentos con menor costo, pero el inconveniente de ser operador dependiente (13). La resonancia magnética abdomino-pélvica se utiliza como método de exploración subsiguiente que permite explorar quistes ováricos endometriósicos y la endometriosis profunda $(2,10)$. La ecoendoscopía rectal es una muy adecuada técnica para observar implantes endometriósicos rectosigmoideos, con una sensibilidad de alrededor del $90 \%$ y una especificidad de hasta el 100\% (10).

El gold standard sigue siendo la laparoscopía diagnóstica, practicando un enfoque sistemático, en búsqueda de adherencias y evaluando la movilidad pélvica; con inspección clara de ovarios, trompas, ligamentos uterosacros, fondo de saco de Douglas, pliegue uterovesical, rectosigmoideo y apéndice $(2,7)$. La experiencia del especialista resulta muy importante para la identificación y el diagnóstico correcto a partir de la inspección visual, con sensibilidad reportada de 94 a $97 \%$ y especificidad de entre 77 y $85 \%$. La endometriosis de tipo superficial suele aparecer típicamente como quemaduras o vesículas de color rojo-marrón, o fuego, con una cierta distorsión anatómica. En los casos más severos, hay más nódulos y placas y la distorsión anatómica es cada vez más evidente (2).

Sin embargo, la laparoscopía diagnóstica plantea el inconveniente de tratarse de un método quirúrgico, por lo que generalmente se decide en un marco preciso, ya sea por tratamiento de infertilidad, síntomas dolorosos, quiste de ovario u otros (2).

Actualmente no hay marcadores en suero que puedan diagnosticar o medir la actividad de la enfermedad en la 
endometriosis. El CA-125 puede verse elevado en enfermedad severa, sin embargo la sensibilidad es muy baja y por lo tanto, no se utiliza de rutina $(13,2)$.

\section{TRATAMIENTO}

Las opciones terapéuticas siempre se deben individualizar en función de la paridad y los deseos de fertilidad de la paciente, la etapa de la enfermedad y los síntomas que se presentan, la edad, las cirugías previas y las preferencias de la paciente (8). Usualmente, lo que se busca es maximizar el uso del tratamiento médico mientras se evita el empleo repetido de cirugías.

En cuanto al tratamiento médico, las drogas que actualmente se utilizan de primera línea para la endometriosis sintomática actúan reduciendo el flujo sanguíneo al útero y bloqueando la función ovárica; esto mediante la creación de un ambiente hipoestrogénico (14).

Se crea una especie de menopausia iatrogénica (5). Aquí se incluyen los anticonceptivos orales combinados (ACO) y las progesteronas, que precisamente poseen la ventaja de estar clasificados dentro del grupo de los tratamientos de bajo costo (14). Lo que se desea con estos fármacos es bloquear la secreción de estrógenos y la actividad de los receptores estrogénicos; así como la activación de los receptores de progesterona.

Debido a que la respuesta de cada individuo a cada droga puede ser distinta, se ha propuesto iniciar de manera gradual; esto significa comenzar con los ACO o las progesteronas de bajo costo y avanzar a otros medicamentos en caso de intolerancia o ineficacia (14). La idea con estos es lograr un control de la enfermedad, pues no ofrecen una cura. Sin embargo, poseen el inconveniente del efecto contraceptivo en mujeres que buscan concebir.

Si fracasa el tratamiento con estrógenosprogestágenos o en caso de que exista alguna contraindicación, se puede plantear la inyección de agonistas de la $\mathrm{GnRH}$ o el tratamiento con danazol. Los agonistas de la $\mathrm{GnRH}$ se pueden utilizar de entrada, pero aún no han demostrado ser más eficaces que los estrógenosprogestágenos. Estos lo que crean es, un ambiente hipoestrogénico dosisdependiente, para inhibir la producción de células endometriales.

Aunque la mayoría del tiempo es posible controlar el dolor y los síntomas producidos por la endometriosis mediante tratamiento médico; hay un remanente de pacientes que pueden permanecer sintomáticas a pesar de la dosificación adecuada de medicamento (8). Para este grupo, la cirugía es la opción siguiente. Puede abarcar desde la laparoscopía simple para el tratamiento superficial, endometriomas o para las lesiones excluidas, hasta procedimientos complejos que incluyen adherenciolisis extensa, ureterolisis, resección parcial de intestino o de vejiga, resección de uréteres $u$ otra que se requiera según el sitio afectado o bien, para tratar la endometriosis profunda.

En general, la evidencia muestra que posterior a un tratamiento quirúrgico por endometriosis, la calidad de vida progresa (principalmente en los casos de endometriosis profunda y endometriosis intestinal) en cuanto a mejoría de síntomas y tratamiento de infertilidad (8). Todavía se requieren más estudios para determinar el impacto en los pacientes con enfermedad mínima solamente. No 
obstante, se debe seguir asesorando a cada paciente sobre las opciones de tratamiento, los riesgos y beneficios del procedimiento quirúrgico; teniendo en cuenta las necesidades individuales de cada una.

Después de la cirugía de una endometriosis profunda 0 de un endometrioma, los estrógenosprogestágenos o los macroprogestágenos pueden disminuir el riesgo de recidiva (15). Estos tratamientos son anticonceptivos y causarán una amenorrea, deben continuarse si no se desea un embarazo, a excepción de los agonistas de la $\mathrm{GnRH}$, cuya prescripción se limita a 6 meses, según lo dictado por The USA Food and Drug Administration (FDA) (3,6). Si se desea tener un embarazo, los antiinflamatorios no esteroideos (AINES) son los únicos fármacos indicados hasta el momento, como analgesia, asociado a la endometriosis $(4,15)$.

Los tratamientos médicos que se consideran emergentes, como los antagonistas de la hormona liberadora de gonadotropina $(\mathrm{GnRH})$, los moduladores selectivos de los receptores estrogénicos (SERMs por sus siglas en inglés), los moduladores selectivos de los receptores de progesterona (SPRMs), los inhibidores de aromatasa, los inmunomuduladores y los anti androgénicos son drogas muy prometedoras $(5,15)$. Sin embargo, algunas manifiestan muchos efectos secundarios, otras presentan la mayoría de estudios en animales y no así en mujeres $y$ algunas otras no han demostrado superioridad (14). Por lo tanto, aún faltan más estudios individuales y comparativos para ponerlos en la práctica diaria.

\section{CONCLUSIÓN}

El diagnóstico de endometriosis no es inusual, al contrario, se realiza en una cantidad considerable de mujeres, la mayoría de estas en edad fértil y con una serie identificada de factores de riesgo modificable y no modificable. La presentación clínica es muy diversa y algunos signos son de mucha utilidad para no demorar la evaluación inicial por endometriosis, mientras que el apoyo en los estudios de imagen la mayoría del tiempo es productivo.

A pesar de que se han planteado tantas teorías para explicar su etiología y ninguna se ha podido probar como definitiva, parece más favorable apegarse a la idea de que realmente se trata de una asociación de todas estas y no una sola que explique todas las presentaciones.

Con frecuencia las pacientes no consumen los tratamientos adecuados, ya sea por ansiedad ante los posibles efectos secundarios, por deseos de embarazo o porque consultan cuando la enfermedad está más evolucionada y les ofrecen pocas opciones conservadoras. Es bien sabido que esto afecta de manera importante en su calidad de vida. Se espera que la investigación futura sobre los mecanismos patológicos de la endometriosis pueda permitir llegar al medicamento ideal, ese que induzca la regresión de las lesiones, proporcione el alivio del dolor y a su vez permita la concepción; al mismo tiempo que disminuya la recurrencia a las cirugías para tratar estos casos. 


\section{REFERENCIAS}

1. Asghari S, Valizadeh A, Aghebati-Maleki L, Nouri M, Yousefi M. Endometriosis: Perspective, lights, and shadows of etiology. Biomedicine \& Pharmacotherapy. 2018 Oct; 106: 163174.https://doi.org/10.1016/i.biopha.2018.06.109

2. Hogg S, Vyas S. Endometriosis update. Obstetrics, Gynaecology \& Reproductive Medicine. 2018 03; 28(3): 61-69. https://doi.org/10.1016/i.ogrm.2017.12.003

3. Patel BG, Lenk EE, Lebovic DI, Shu Y, Yu J, Taylor RN. Pathogenesis of endometriosis: Interaction between endocrine and inflammatory pathways. Best Practice \& Research Clinical Obstetrics \& Gynaecology. 2018 07; 50: 50-60. https://doi.org/10.1016/j.bpobgyn.2018.01.006

4. Tomassetti C, D'Hooghe T. Endometriosis and infertility: Insights into the causal link and management strategies. Best Practice \& Research Clinical Obstetrics \& Gynaecology. 2018 08; 51: 2533. https://doi.org/10.1016/..bpobgyn.2018.06.002

5. Clemenza S, Sorbi F, Noci I, Capezzuoli T, Turrini I, Carriero C, Buffi N, Fambrini M, Petraglia F. From pathogenesis to clinical practice: Emerging medical treatments for endometriosis. Best Practice \& Research Clinical Obstetrics \& Gynaecology. 2018 08; 51: 92-101.https://doi.org/10.1016/i.bpobgyn.2018.01.021

6. Chauvet $\mathrm{P}$, Gremeau A, Roman H, Canis M, Rabischong B, Pouly J, Bourdel N. Cirugía de la endometriosis anexial. EMC - Ginecología-Obstetricia. 2017 06; 53(2): 1-11.https://doi.org/10.1016/s1283-081x(17)842867

7. Shafrir A, Farland L, Shah D, Harris H, Kvaskoff M, Zondervan K, Missmer S. Risk for and consequences of endometriosis: A critical epidemiologic review. Best Practice \& Research Clinical Obstetrics \& Gynaecology. 2018 08; 51: 1-15. https://doi.org/10.1016/j.bpobgyn.2018.06.001

8. Arcoverde FVL, Andres MDP, Borrelli GM, Barbosa PDA, Abrão MS, Kho RM. Surgery for Endometriosis Improves Major Domains of Quality of Life: A Systematic Review and Meta-Analysis. Journal of Minimally Invasive Gynecology. 2019 02; 26(2): 266-278.https://doi.org/10.1016/.jimig.2018.09.774

9. Leone Roberti Maggiore U, Inversetti A, Schimberni M, Viganò P, Giorgione V, Candiani M. Obstetrical complications of endometriosis, particularly deep endometriosis. Fertility and Sterility. 2017 Dec; 108(6): 895-912. https://doi.org/10.1016/i.fertnstert.2017.10.035

10. Thomassin-Naggara I, Fedida B, Ballester M, Bazot M, Daraï E. Diagnóstico por imagen de la endometriosis pélvica. EMC - Ginecología-Obstetricia. 2017 09; 53(3): 1-10.https://doi.org/10.1016/s1283-081x(17)85962$\underline{2}$

11. Sauvanet E, Petit E, Mephon A. Endometriosis genital y extragenital. EMC - Tratado de Medicina. 201806 ; 22(4): 1-8. https://doi.org/10.1016/s1636-5410(18)41301-3

12. Gordts S, Koninckx P, Brosens I. Pathogenesis of deep endometriosis. Fertility and Sterility. 2017 Dec; 108(6): 872-885. https://doi.org/10.1016/j.fertnstert.2017.08.036

13. DF, Flores I, Waelkens E, D'Hooghe T. Noninvasive diagnosis of endometriosis: Review of current peripheral blood and endometrial biomarkers. Best Practice \& Research Clinical Obstetrics \& Gynaecology. 2018 07; 50: 72-83. https://doi.org/10.1016/j.bpobgyn.2018.04.001

14. Vercellini P, Buggio L, Frattaruolo MP, Borghi A, Dridi D, Somigliana E. Medical treatment of endometriosisrelated pain. Best Practice \& Research Clinical Obstetrics \& Gynaecology. 2018 08; 51: 6891. https://doi.org/10.1016/.j.bpobgyn.2018.01.015

15. Azoulay C, Bardy C, Lasserre M, Brun J. Tratamientos farmacológicos de la endometriosis (a excepción de la adenomiosis). EMC - Ginecología-Obstetricia. 2018 03; 54(1): 1-15.https://doi.org/10.1016/s1283$081 \times(18) 88083-3$ 